Hautarzt 2022 $\cdot 73: 560-562$

https://doi.org/10.1007/s00105-021-04899-x

Angenommen: 15. September 2021

Online publiziert: 11. Oktober 2021

(c) Der/die Autor(en) 2021

\section{Bullöses Pemphigoid mit Aussparung eines Armes nach axillärer Lymphadenektomie}

\author{
S. Janßen · B. Homey · T. M. Jansen \\ Klinik für Dermatologie, Universitätsklinikum Düsseldorf, Düsseldorf, Deutschland
}

\title{
Zusammenfassung
}

Wir präsentieren den Fall einer 83-jährigen Patientin mit einem bullösen Pemphigoid (BP) unter Aussparung des linken Armes bei Zustand nach axillärer Lymphadenektomie nach Mammakarzinom. Lokalisierte Formen des bullösen Pemphigoids treten v. a. bei Lymphödemen oder nach Radiatio auf. Das Fehlen des Blasenbefalls nach einer Lymphadenektomie stellt eine seltene und interessante Manifestation dar. Pathophysiologisch könnte durch den Rückgang der T-Zell-Aktivierung bei fehlenden peripheren Lymphknoten und somit vermindertem entzündlichem Infiltrat die Blasenbildung abgeschwächt sein bzw. vollkommen ausbleiben.

\section{Schlüsselwörter}

Blasenbildende Dermatosen · Autoimmundermatosen · Lymphknotendissektion · T-Zellvermittelte Dermatosen · Lymphknoten

Die Abb. 1 wurde 2020 bereits im Rahmen einer Posterpräsentation veröffentlicht.

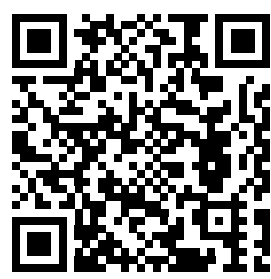

QR-Code scannen \& Beitrag online lesen
Das bullöse Pemphigoid ist eine blasenbildende Autoimmundermatose insbesondere des älteren Menschen, die mit einer subepidermalen Spaltbildung durch Bildung von Autoantikörpern gegen hemidesmosomale Antigene (BP230, BP180) einhergeht. Es handelt sich um eine Dermatose mit exanthematischem bzw. symmetrischem oder lokalisiertem Befall. Die lokalisierte Form des bullösen Pemphigoids ist selten und wird in Fallberichten beispielsweise nach Radiatio oder im Bereich von Lymphödemen beschrieben [1-3]. Wir berichten über den seltenen Fall eines bullösen Pemphigoids mit Aussparung des linken Armes nach totaler Lymphadenektomie bei Zustand nach Mammakarzinom.

\section{Falldarstellung}

Eine 83-jährige Patientin stellte sich mit progredienten bullösen Hautveränderungen im Bereich des gesamten Integuments mit Aussparung des linken Armes in unserer Ambulanz vor. Diese seien vor ungefähr 4 Wochen aufgetreten und mit starkem Pruritus einhergehend. An Vorerkrankungen war bei der Patientin ein Mammakarzinom bekannt, welches vor 18 Jahren mittels Mastektomie, totaler Lymphadenektomie, Radio- und Chemotherapie behandelt wurde. Im Anschluss sei die Patientin in engmaschiger gynäkologischer Nachsorge vorstellig gewesen. Die weitere Anamnese ergab, dass die Patientin zudem an einer arteriellen Hypertonie, einem Diabetes mellitus Typ 2, einer chronischen Niereninsuffizienz sowie einer monoklonalen Gammopathie litt.

\section{Klinischer Befund}

Bei der körperlichen Untersuchung imponierten am rechten Unterarm, an der rechten Axilla, an beiden Oberschenkeln sowie im Brustbereich multiple, bis zu $2 \mathrm{~cm}$ durchmessende, prall gefüllte Bullae auf erythematösem Grund ( $\bullet$ Abb. 1a).Zudem zeigten sich links thorakal eine $10 \mathrm{~cm}$ messende strichförmige reizlose Narbe bei Zustand nach Ablatio mammae sowie links axillär eine $6 \mathrm{~cm}$ messende reizlose Cicatrix bei Zustand nach totaler Lymphadenektomie. Im Bereich des linken Armes zeigten sich keine der genannten Hautveränderungen, auch die linke Axilla blieb ausgespart (•Abb. 1b, c). Es zeigten sich keine Anzeichen eines Lymphödems des linken 


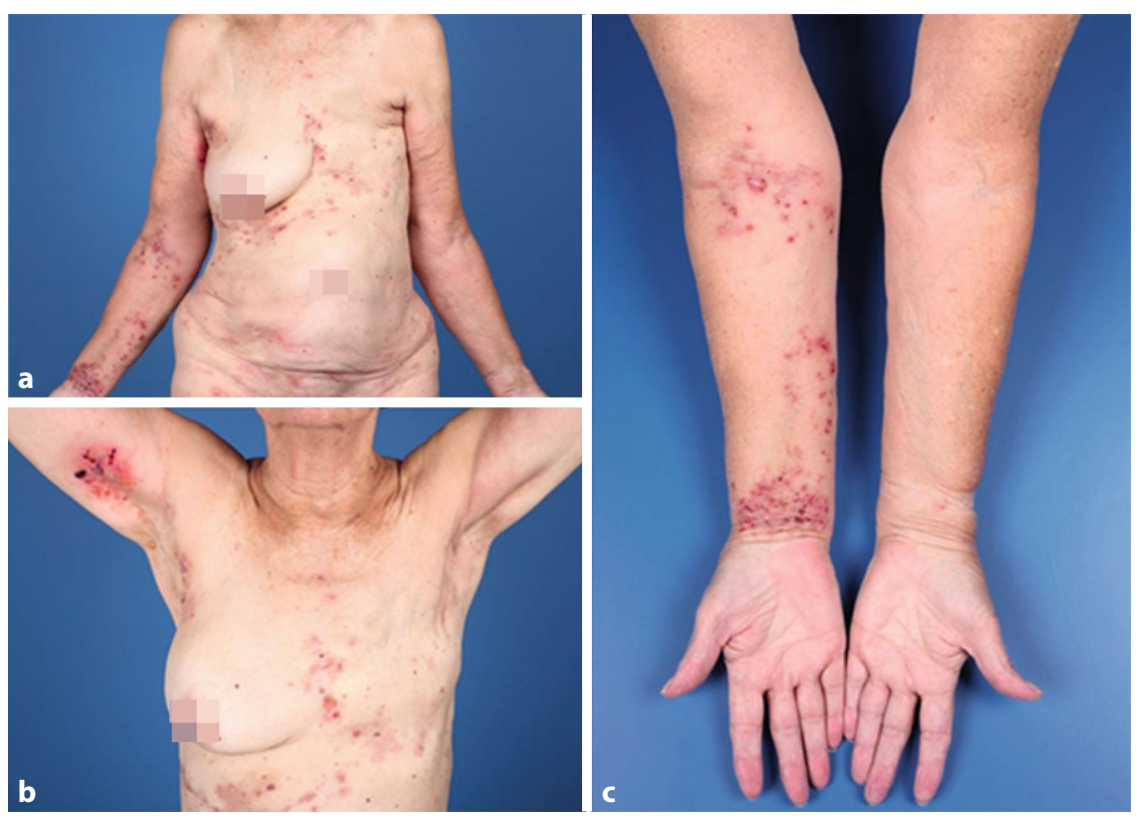

Abb. $1 \Delta$ a Im Bereich des rechten Armes sowie im Brustbereich zeigen sich multiple 1-2 cm durchmessende Bullae auf erythematösem Grund. b Aussparung der linken Axilla. c Aussparung des linken Armes

Armes. Die Patientin gab starken Pruritus (NRS 8/10) sowie eine allgemeine Abgeschlagenheit an. Der Juckreiz trat v.a. an den Oberschenkeln sowie am rechten Handgelenk auf, am linken Arm war die Patientin beschwerdefrei.

\section{Diagnose}

Laborchemisch auffällig waren ein leicht erhöhtes CRP sowie Kreatinin bei bekannter Niereninsuffizienz. Im Differenzialblutbild imponierte eine relative Eosinophilie. In der dermatohistopathologischen Untersuchung einer Stanzbiospie des rechten medialen Oberschenkels von läsional zeigte sich eine subepidermale Blasenbildung mit eosinophilenreichem Entzündungsinfiltrat. In der direkten Immunfluoreszenzuntersuchung von periläsional fanden sich lineare Ablagerungen von AntilgG-Antikörpern entlang der Basalmembranzone, die indirekte Immunfluoreszenz wies BP180- und BP230-Autoantikörper nach (Biochip-Mosaik-Diagnostik).

\section{Therapie und Verlauf}

Wir initiierten eine systemische Therapie mit Doxycyclin $200 \mathrm{mg}$ p.o. und Prednisolon $40 \mathrm{mg}$ p.o. täglich (0,5 mg Prednisolon/kg Körpergewicht) in ausschleichen- der Dosierung mit einer Reduktion von $10 \mathrm{mg}$ pro Woche. Zudem erfolgte eine topische Therapie mittels Clobetasolpropionat-haltigen Externa, die wir im Verlauf auf Mometasonfuroat-haltige Externa umstellten. Darunter zeigte sich der Hautbefund langsam regredient. Die Patientin wurde mit Doxycyclin und Prednisolon $20 \mathrm{mg}$ entlassen und stellte sich im Verlauf nicht erneut in unserer Ambulanz vor.

\section{Diskussion}

Lokalisierte Formen des bullösen Pemphigoids sind in der Literatur selten, es handelt sich v.a. um Hautbefunde im Bereich von Lymphödemen oder nach stereotaktischer Bestrahlung. Im Fall eines Lymphödems wird das lokalisierte Auftreten mit der Spaltung der Epidermisverbindung aufgrund eines erhöhten hydrostatischen Drucks sowie der erhöhten Kapillardurchlässigkeit und dem vermehrten Austreten von Immunmediatoren erklärt [2]. Das BP nach Radiatio tritt am häufigsten bei Patientinnen mit Mammakarzinomen auf [3]. Pathophysiologisch wird angenommen, dass durch die strahleninduzierte strukturelle Veränderung der Basalmembranzone die Produktion von Autoantikörpern verstärkt wird und eine Hemmung der T-Zell-Suppressor-Aktivität induziert wird.
Zudem werden durch eine Radiatio die Spiegel von MMP9 (Matrix-Metallopeptidase 9) und VEGF beeinflusst, welche eine erhöhte Expression in läsionaler Haut beim BP aufweisen [3].

Der Befund eines bullösen Pemphigoids mit der Aussparung einer Extremität nach Lymphadenektomie wurde in der Literatur lediglich 1-mal beschrieben. Dieser Fall berichtet über den Kasus einer 74jährigen Patientin mit einem generalisierten BP und Aussparung des linken Beines, das, anders als in unserem Fall, durch ein Lymphödem als Folge einer Lymphknotendissektion gekennzeichnet war. Die Autoren stellten hier die Annahme eines Rückgangs der lokalen zellulären Immunität mit verminderter T-Zell-Aktivierung [4]. Auch in diesem Fall war der Pruritus an dem von Blasen ausgesparten Bein nicht vorhanden. In der Biopsie der nicht betroffenen Haut zeigte sich kein inflammatorisches Infiltrat, jedoch waren lineare IgG-Ablagerungen sichtbar [4].

Pathophysiologisch kommt es nach Bindung der Antikörper zur Freisetzung von Interleukinen (v.a. IL-6, IL-8) aus Keratinozyten, zur Komplementaktivierung sowie zur Bildung eines inflammatorischen Infiltrats aus Neutrophilen, Eosinophilen und Makrophagen in der oberen Dermis. Durch Freisetzung reaktiver Sauerstoffspezies und Proteinasen an der dermoepidermalen Junktionszone kommt es zur Spaltbildung zwischen Dermis und Epidermis [5]. T-Zellen gelten zunehmend als Schlüsselakteure in der Pathogenese autoimmunblasenbildender Dermatosen und bewirken u. a. eine Aufrechterhaltung der Entzündung und Verstärkung des Gewebeschadens. Beim BP zeigt sich eine erhöhte Zahl an Th2-Zellen und IL-4, die die Immunantwort, Antikörperproduktion sowie B-Zell-Proliferation fördert. Durch die Sekretion von IL-17A aus pathogenen Th17-Zellen wird die Entzündungsreaktion über die Aktivierung von Neutrophilen verstärkt und der Gewebeschaden erhöht $[5,6]$.

Unser Fall deutet an, dass durch fehlende axilläre Lymphknoten die T-Zell-Aktivierung sowie die Interleukinfreisetzung so vermindert werden, dass sich klinisch auch trotz Vorhandensein von z. B. einzelnen kubitalen Lymphknoten, die im Vergleich zur Axilla eine geringe Anzahl darstellen, keine 
Blasenbildung manifestiert. Dadurch, dass in unserem Fall keine Biopsie im Bereich der nichtbetroffenen Haut durchgeführt wurde, bleibt diese Hypothese spekulativ.

Tierexperimentelle Studien der letzten Jahre zeigen, dass nach Lymphknotenexstirpation die T-Zell-abhängige Kontakthypersensitivitätsreaktion in der ipsilateralen Extremität signifikant reduziert ist [7]. Dies könnte darauf hinweisen, dass der regionale Lymphknoten für periphere T-Zellvermittelte Hautreaktionen entscheidend ist, um z. B. die Population und Rekrutierung von "tissue resident memory T-cells" (Trm) in der Haut zu kontrollieren und in der Peripherie aufzubauen. Hier kommt dem Lymphknoten möglicherweise eine entscheidende Funktion in der Prägung und Aufrechterhaltung dieser Population zu [8].

Zusammenfassend ruft dieser eindrucksvolle klinische Fall immunologisch wichtige Hypothesen hervor und weist auf die entscheidende Rolle des peripheren Lymphknotens in der lokalen Immunantwort bei blasenbildenden Dermatosen hin.

\section{Fazit für die Praxis}

- Neben dem exanthematischen und lokalisierten Auftreten des bullösen Pemphigoids zeigt unser Fall die einseitige Aussparung des Krankheitsbildes nach Lymphadenektomie.

- Für die klinische Ausbildung autoimmunblasenbildender Dermatosen kommt den peripheren Lymphknoten und ihrer Interaktion mit Immunzellen womöglich eine maßgebliche Rolle zu. Zur näheren Charakterisierung des Lymphknotens in T-Zell-vermittelten Hauterkrankungen bedarf es weiterer klinischer Beobachtung und Forschung.

\section{Korrespondenzadresse}

\section{S. Janßen}

Klinik für Dermatologie, Universitätsklinikum Düsseldorf

Moorenstr. 5, 40225 Düsseldorf, Deutschland sarah.janssen@med.uni-duesseldorf.de

Funding. Open Access funding enabled and organized by Projekt DEAL.

\section{Bullous pemphigoid with unilateral sparing after axillary lymphadenectomy}

An 83-year-old woman presented to our outpatient clinic with bullous pemphigoid with a unilateral sparing of the left arm after axillary lymphadenectomy because of breast cancer. Cases of localized manifestations of bullous pemphigoid are mainly caused by lymphedema or radiation. The absence of blistering after lymphadenectomy is a rare and interesting manifestation. Pathophysiologically, blister formation may be attenuated or absent altogether due to decreased T-cell activation and thus reduced inflammatory infiltrate because of the absence of peripheral lymph nodes.

\section{Keywords}

Bullous diseases - Autoimmune diseases · Lymph node dissection · T-cell-mediated dermatoses . Lymph node

\section{Einhaltung ethischer Richtlinien}

Interessenkonflikt. S. Janßen, B. Homey und T.M.Jansen geben an, dass kein Interessenkonflikt besteht.

Für diesen Beitrag wurden von den Autoren keine Studien an Menschen oder Tieren durchgeführt. Für die aufgeführten Studien gelten die jeweils dort angegebenen ethischen Richtlinien. Für Bildmaterial oder anderweitige Angaben innerhalb des Manuskripts, über die Patienten zu identifizieren sind, liegt von ihnen und/oder ihren gesetzlichen Vertretern eine schriftliche Einwilligung vor.

Open Access. Dieser Artikel wird unter der Creative Commons Namensnennung 4.0 International Lizenz veröffentlicht, welche die Nutzung, Vervielfältigung, Bearbeitung, Verbreitung und Wiedergabe in jeglichem Medium und Format erlaubt, sofern Sie den/die ursprünglichen Autor(en) und die Quelle ordnungsgemäßnennen, einen Link zur Creative Commons Lizenz beifügen und angeben, ob Änderungen vorgenommen wurden.

Die in diesem Artikel enthaltenen Bilder und sonstiges Drittmaterial unterliegen ebenfalls der genannten Creative Commons Lizenz, sofern sich aus der Abbildungslegende nichts anderes ergibt. Sofern das betreffende Material nicht unter der genannten Creative Commons Lizenz steht und die betreffende Handlung nicht nach gesetzlichen Vorschriften erlaubt ist, ist für die oben aufgeführten Weiterverwendungen des Materials die Einwilligung des jeweiligen Rechteinhabers einzuholen.

Weitere Details zur Lizenz entnehmen Sie bitte der Lizenzinformation auf http://creativecommons.org/ licenses/by/4.0/deed.de.

\section{Literatur}

1. Binitha MP, Vishnu VV, Sreekanth S, Reena Mariyath OK (2014) Localized bullous pemphigoid on sites of radiotherapy and lymphedema in the same patient. Indian Dermatol Online J 5:101-103

2. Perez A, Clements SE, Benton E et al (2009) Localized bullous pemphigoid in a patient with primary lymphoedema tarda. Clin Exp Dermatol 34:e931-e933

3. Mul VE, van Geest AJ, Pijls-Johannesma MC et al (2007) Radiation-induced bullous pemphigoid: a systematic review of an unusual radiation side effect. Radiother Oncol 82(1):5-9

4. Roguedas AM, Crespel E, Kupfer I et al (2006) Bullous pemphigoid sparing acquired lymphedema. Ann Dermatol Venereol 133:250-252

5. Sadik CD, Schmidt E (2019) Resolution in bullous pemphigoid. Semin Immunopathol 41:645-654

6. Fang H, Li Q, Wang G (2020) The role of T cells in pemphigus vulgaris and bullous pemphigoid. Autoimmun Rev 19:102661

7. García Nores GD, Ly CL, Savetsky IL et al (2018) Regulatory $\mathrm{T}$ cells mediate local immunosuppression in lymphedema. J Invest Dermatol 138:325-335

8. BeuraLK, WijeyesingheS, ThompsonEA etal (2018) $\mathrm{T}$ cells in non-lymphoid tissues give rise to lymph node resident memory T cells. Immunity 48:327 